\title{
Reorientation in a Two-Dimensional Environment: II. Do Pigeons (Columba livia) Encode the Featural and Geometric Properties of a Two-Dimensional Schematic of a Room?
}

\author{
Debbie M. Kelly and Marcia L. Spetch \\ University of Alberta
}

\begin{abstract}
Pigeons (Columba livia) searched for a hidden target area in images showing a schematic rectangular environment. The absolute position of the goal varied across trials but was constant relative to distinctive featural cues and geometric properties of the environment. Pigeons learned to use both of these properties to locate the goal. Transformation tests showed that pigeons could use either the color or shape of the features, but performance was better with color cues present. Pigeons could also use a single featural cue at an incorrect corner to distinguish between the correct corner and the geometrically equivalent corner; this indicates that they did not simply use the feature at the correct corner as a beacon. Interestingly, pigeons that were trained with features spontaneously encoded geometry. The encoded geometric information withstood vertical translations but not orientation transformations.
\end{abstract}

Many organisms are faced with the task of navigating within an environment. This task may require the ability to specifically remember a particular location such as a nest site. A great deal of research has been conducted to understand what mechanisms and environmental properties allow animals to navigate accurately (for a review, see Healy, 1998). Many studies have shown that visual landmarks are an important source of information and are used by many different species for navigation within a familiar environment (for a review, see Cheng \& Spetch, 1998). In addition, several studies have revealed that the geometry defined by continuous surfaces can also be an important cue for an animal to obtain a sense of bearing for orientation (Cheng, 1986; Cheng \& Gallistel, 1984; Gallistel, 1990; Margules \& Gallistel, 1988).

Cheng's (1986) experiments with rats provided the initial impetus for studying whether and how animals use the overall shape, or geometry, of their environment for orienting. In a reference memory experimental task, Cheng trained rats to find food hidden in one corner of a fully enclosed rectangular environment. The orientation of the enclosure in the room varied across trials. One of four very distinct featural cues was placed in each corner of the enclosure. Cheng hid food in a glass bottle in front of one of the featural cues (the cue was counterbalanced across subjects), placed a rat in the enclosure, and allowed it to search among the bottles

Debbie M. Kelly and Marcia L. Spetch, Department of Psychology, University of Alberta, Edmonton, Alberta, Canada.

This research was supported by a Natural Sciences and Engineering Research Council of Canada research fellowship to Debbie M. Kelly and grant to Marcia L. Spetch. The preparation of this article was further supported by National Institute of Mental Health Grant MH61810 to Alan C. Kamil, who provided postdoctoral funding to Debbie M. Kelly. We thank W. F. Bischof for assistance in writing the analyses programs and M. Schulze for assistance with conducting the experiments.

Correspondence concerning this article should be addressed to Debbie M. Kelly, who is now at the Department of Psychology, University of Saskatchewan, 172.2 Arts Building, 9 Campus Drive, Saskatoon, Saskatchewan S7N 5A5, Canada. E-mail: Debbie.Kelly@duke.usask.ca to find the one containing food, which was in the same corner on every trial. The rat was trained until it chose the correct corner first on 9 of 10 consecutive trials. Although the rats could easily have solved the task by encoding the distinctive featural cue in the same corner as the hidden food, the rats made several errors. In examining the errors, Cheng found that the rats made significantly more errors to the corner diagonally opposite from the correct corner than would be expected compared with an even distribution of errors. Cheng termed these systematic rotational errors because if the rats encoded only the geometry of the environment, the correct corner would be indistinguishable from the diagonally opposite corner; the two corners would both be geometrically correct.

Following Cheng's (1986) study, several researchers examined how other species use geometric and featural information to reorient within a rectangular enclosure. Interestingly, many other species show the ability to use the geometric properties of the environment and the featural cues contained within the environment (e.g., fish: Sovrano, Bisazza, \& Vallortigara, 2002, 2003; pigeons: Kelly, Spetch, \& Heth, 1998; chicks: Vallortigara, Zanforlin, \& Pasti, 1990; rhesus monkeys: Gouteux, Thinus-Blanc, \& Vauclair, 2001; and adult humans: Hermer \& Spelke, 1994, 1996; see also Wang \& Spelke, 2002). However, the extent to which featural and geometric information is relied on and how much of the available information is used seems to differ across species and with experience.

Kelly et al. (1998) examined the encoding of featural and geometric information by pigeons by means of a paradigm similar to Cheng's (1986). Two groups of pigeons were trained on a reference memory task to find food hidden in one of four visually identical containers located in the corners of a fully enclosed rectangular environment. The position of the reinforced container was counterbalanced across subjects. One group of birds was initially trained with distinct featural information at each corner of the environment, whereas the second group was trained without any distinct features present (i.e., only the geometric properties of the environment were available). Similar to previous research with 
chicks (Vallortigara et al., 1990), Kelly et al. found that pigeons trained with the features present nevertheless encoded the geometry of the environment: When the researchers removed all of the distinctive featural information, these pigeons distributed most of their choices between the two geometrically correct corners. Interestingly, the researchers also found that the initial training experience was an important determinant of subsequent reliance on geometric and featural information. The pigeons in the geometry group were retrained with the distinctive featural cues. Upon completion of retraining, the researchers conducted an affine transformation test with both groups of pigeons. This test pits geometric and featural information against each other by moving each feature one position either clockwise or counterclockwise (i.e., the features that were in geometrically correct corners were moved to geometrically incorrect corners). The birds initially trained with features showed strong control by the correct feature and continued to choose the container associated with this feature even though it was now located in a geometrically incorrect corner. However, the birds initially trained with geometry divided their choices between the two geometrically correct corners and the featurally correct corner. This result shows that the birds' initial experience in the environment influenced how they later relied on both geometric and featural information.

To date, all of these studies examining the encoding of featural and geometric information by animals have used a navigable open-field task. However, a touch-screen task conducted within a standard operant chamber has been successfully used to study how animals, and in particular birds, use landmarks and landmark configurations (e.g., Spetch, 1995; Spetch, Cheng, \& MacDonald, 1996; Spetch et al., 1997). To perform this task, animals view a "spatial arena" on a computer monitor with an attached touch frame. Images are presented on the screen with various graphic stimuli serving as landmarks. The animal's task is to use these landmarks to locate and respond directly to a small unmarked area that serves as the goal. If the animal accurately responds in the location of the goal, the animal is reinforced by limited access to a feeder usually located near the viewing screen. It is quite apparent that the touch-screen task and the open-field task differ in many ways (see Spetch \& Kelly, in press, for a discussion of the task differences), yet processes of landmark-based searching have shown remarkable generality. To date, however, no studies have examined whether birds encode geometric and featural information in a similar way within a two-dimensional (2-D) touch-screen task as has been shown in a three-dimensional (3-D) rectangular room.

We initially addressed the question of whether the encoding of geometric and featural information in a 2-D task would be similar to that seen in 3-D tasks by examining how adult humans use these types of cues in a schematic representation of a room (Kelly \& Spetch, 2004). Participants viewed a schematic representation of a rectangular environment displayed in various orientations on a touch-screen monitor. The participants earned points for touching the correct corner of the rectangle (with the correct corner counterbalanced across participants). One group of participants was provided with distinct featural cues adjacent to each corner of the environment. The second group was shown the environment without any distinctive features and therefore had to rely on the geometric properties of the rectangle to solve the task. Whereas almost all of the participants who were provided with distinctive featural cues readily learned the task, only some of the participants provided with geometric cues alone were able to learn to select only the two geometrically correct corners. However, when the featurally trained participants were presented with test images in which all of the features were removed, they did not respond randomly; rather they divided their choices between the two geometrically correct corners. This result showed that the featurally trained participants had encoded the geometric information even though this was not needed to solve the task; furthermore, these participants did not respond differently than those who had initially learned the task on the basis of only geometry. Thus, even though learning about the features in this 2-D task was easier than learning about the geometry, the participants showed conjoining of geometric and featural cues-a consistent result shown by adult humans in a 3-D environment (both navigable: Hermer \& Spelke, 1994, 1996; and nonnavigable: Gouteux, Vauclair, \& ThinusBlanc, 2001).

In the current study, we investigated how pigeons encoded featural and geometric information when presented with a 2-D schematic of a rectangular environment. The results of this study were of interest for several reasons. First, a comparison of results from this spatially limited environment with our previous investigations of featural and geometric encoding in a 3-D navigable environment allowed us to examine how the nature and scale of the spatial environment influence geometric and featural encoding by pigeons. Second, this study complements previous investigations that have examined use of discrete landmarks for spatial search within 2-D touch-screen environments. One potentially important feature of these previous touch-screen studies is that the orientation of the landmark array was always held constant so that external screen or chamber cues provided a directionally stable frame of reference. The current study differed from these previous landmark studies in that we explored use of both geometric and featural cues, and we rotated the rectangular environment across trials so that the pigeons could not use the stable extraenvironmental cues to locate the correct corner. Whether pigeons could acquire the task was also an important question. Finally, we were interested in whether pigeons' use of geometric and featural information in a 2-D environment would be similar to that shown by humans. Although some processes of landmark-based searching seem to be general across pigeons and humans (Spetch, 1995), landmark array expansion tests have revealed that humans and pigeons sometimes use quite different strategies (Spetch et al., 1996, 1997). In particular, adult humans spontaneously adopt a relational rule that holds across scale transformations, a strategy that might reflect experience with map or model representations of space. Thus, it is of interest to see how pigeons would compare with humans in their ability to extract geometry from a schematic representation of a room.

In Experiment 1A, we examined whether and how pigeons conjoin geometric and featural information presented in a schematic representation of a rectangular environment. By initially training some pigeons with geometry only and some with both features and geometry, we examined whether geometry is spontaneously encoded even when features are present. By subsequently training all pigeons on the reverse condition, we could assess whether training order affected the information encoded. Through a series of test trials that followed each training condition, we examined (a) the influence of general environmental cues (i.e., 
background shading and absolute position of the environment on the screen), (b) the influence of the distinctive features (i.e., the color or shape of the features and how many features were present), and (c) whether geometric information was encoded and whether the geometric representation could withstand rotations. In Experiment 1B, we gave the pigeons an additional series of tests to further examine the encoding of individual features and the relative dominance of featural and geometric information.

\section{Experiment 1A}

\section{Method}

\section{Subjects}

The subjects were 8 Silver King pigeons (Columba livia), all experimentally naïve with spatial search tasks. We maintained the pigeons at $85 \%$ free-feeding weight with mixed grain during experimental sessions and with supplemental feedings of Kay Tee pigeon pellets. The birds were housed in large individual cages under a 12-hr light-dark cycle (with light onset at 6:00 a.m.). Grit and water were provided ad lib in the home cages. Four pigeons were randomly assigned to group Feature-Geometry (F-G) and 4 to group Geometry-Feature (G-F). Within each group, each pigeon was assigned to a different rotation subgroup (see explanation in the Design section). Within each subgroup, the corner of the rectangle that was designated as correct was counterbalanced across subjects.

\section{Apparatus}

The experiment was conducted using three large custom-built operant chambers. Two of the chambers' dimensions (height $\times$ depth $\times$ width) were $42 \times 32 \times 73 \mathrm{~cm}$ and one was $36 \times 34 \times 50 \mathrm{~cm}$. A Zenith 1492 color monitor with an attached infrared touch frame (1492 Smart Frame; Carroll Touch, Round Rock, TX) was placed against the opening at the back of each chamber. The touch frame was recessed $3 \mathrm{~cm}$ from the opening and $1.6 \mathrm{~cm}$ from the monitor. A jagged Plexiglas edge was mounted to the front of the recessed ledge to discourage pigeons from perching on the touch frame. Food rewards were presented using two standard pigeon feeders, one located on each side of the monitor on the back wall of the larger chambers or on the sidewalls of the smaller chamber. The touch frame was programmed to detect individual responses (i.e., it detected when an infrared beam was broken and then had to return to an unbroken state before another response could be recorded). Mounted inside each feeder was a small lamp that illuminated the feeder when a reward was available. An infrared beam measured head entries into the feeders and was used to limit food access. The chambers were connected to microcomputers located in an adjacent room. These computers controlled all of the experimental contingencies and recorded responses.

\section{Images}

All images presented a rectangular environment centered on a white background. (Many of the images used were identical to the images presented to human participants in Kelly \& Spetch, 2004.) Each corner of the rectangular environment contained a black response square $(0.5 \times 0.5$ $\mathrm{cm}$ ), and up to four uniquely shaped features (approximately $0.5 \times 0.5 \mathrm{~cm}$, depending on the type of feature present) were presented adjacent to the corners. Because the number and type of features presented varied across training and testing sessions, they are explained in more detail in the Feature training and Feature testing sections. The gray shading defining the rectangle was $2 \times 4 \mathrm{~cm}$.

\section{Design}

The experiment was a mixed-factor design (see Table 1). The first between-subjects factor was training order with two levels: groups F-G and G-F. The pigeons in group F-G were trained first in the feature condition and then received the following tests: identical, all correct, color-only, shape-only, distant, move, and no shading (see Table 2). They were then retrained in the geometry condition and received the following tests: identical and new rotation. The pigeons in group G-F were trained first in the geometric condition and then received the following tests: identical, move, no shading, and new rotation. They were then retrained in the feature condition and received the following tests: identical, all correct, color-only, shape-only, and distant (see Table 2).

The second between-subjects factor was an image rotation subgroup with four levels: $0^{\circ}-180^{\circ}, 45^{\circ}-225^{\circ}, 90^{\circ}-270^{\circ}$, and $135^{\circ}-315^{\circ}$. Two pigeons in each of groups F-G and G-F were assigned to each subgroup, and these subgroups differed in which image rotations were experienced during training and testing. A total of eight different image rotations were used: $0^{\circ}, 45^{\circ}, 90^{\circ}, 135^{\circ}, 180^{\circ}, 225^{\circ}, 270^{\circ}$, and $315^{\circ}$, but each pigeon was trained with only six of them. The subgroup name indicates the rotations that were omitted in training. For example, pigeons in the subgroup $0^{\circ}-180^{\circ}$ were trained with all the rotations except $0^{\circ}$ and $180^{\circ}$, whereas pigeons in the subgroup $45^{\circ}-225^{\circ}$ were trained with all the rotations except $45^{\circ}$ and $225^{\circ}$. We adopted this procedure to (a) examine performance on novel rotations and (b) allow us to make comparisons with the human participants we examined using similar procedures in a previous study (Kelly \& Spetch, 2004).

\section{Training and Testing Procedures}

Preliminary training. All pigeons received several sessions of training with a modified autoshaping procedure to establish reliable pecking at the monitor. Initially, a $0.5-\mathrm{cm}$ black square was intermittently presented in various screen positions against a white background, with 60 -s intertrial intervals (ITIs). The black square remained on until the bird pecked at the square or $8 \mathrm{~s}$ elapsed, and then food was presented. Once reliable pecking

Table 1

Design of Experiment $1 A$

\begin{tabular}{|c|c|c|c|c|c|c|c|}
\hline \multicolumn{4}{|c|}{ Group F-G } & \multicolumn{4}{|c|}{ Group G-F } \\
\hline Rotation & Rotation & Rotation & Rotation & Rotation & Rotation & Rotation & Rotation \\
\hline $0^{\circ}-180^{\circ}$ & $45^{\circ}-225^{\circ}$ & $90^{\circ}-270^{\circ}$ & $135^{\circ}-315^{\circ}$ & $0^{\circ}-180^{\circ}$ & $45^{\circ}-225^{\circ}$ & $90^{\circ}-270^{\circ}$ & $135^{\circ}-315^{\circ}$ \\
\hline \multicolumn{4}{|c|}{ Feature training } & \multicolumn{4}{|c|}{ Geometric training } \\
\hline \multicolumn{4}{|c|}{ Feature testing } & \multicolumn{4}{|c|}{ Geometric testing } \\
\hline \multicolumn{4}{|c|}{ Geometric retraining } & \multicolumn{4}{|c|}{ Feature retraining } \\
\hline \multicolumn{4}{|c|}{ Geometric testing } & \multicolumn{4}{|c|}{ Feature testing } \\
\hline
\end{tabular}

Note. $\mathrm{F}=$ feature; $\mathrm{G}=$ geometry. 
Table 2

Summary of Experimental Testing Conditions

\begin{tabular}{|c|c|c|}
\hline \multirow[b]{2}{*}{ Experiment and group } & \multicolumn{2}{|c|}{ Test } \\
\hline & Feature & Geometric \\
\hline \multicolumn{3}{|l|}{$1 \mathrm{~A}$} \\
\hline \multirow[t]{9}{*}{ Group F-G } & Identical-before & Identical-before \\
\hline & All correct-before & New rotation \\
\hline & Color-only & Identical-after \\
\hline & Shape-only & \\
\hline & Distant & \\
\hline & Move & \\
\hline & No shading & \\
\hline & Identical-after & \\
\hline & All correct-after & \\
\hline \multirow[t]{7}{*}{ Group G-F } & Identical-before & Identical-before \\
\hline & All correct-before & New rotation \\
\hline & Color-only & Move \\
\hline & Shape-only & No shading \\
\hline & Distant & Identical-after \\
\hline & Identical-after & \\
\hline & All correct-after & \\
\hline \multirow[t]{4}{*}{ IB } & Affine-clockwise & \\
\hline & Affine-counterclockwise & \\
\hline & Distant-near & \\
\hline & Distant-far & \\
\hline
\end{tabular}

Note. The identical test and the all correct tests were conducted both before and after the feature tests. $F=$ feature; $G=$ geometry.

was established, the ITI was reduced to $1 \mathrm{~s}$ and the black square remained on until the bird pecked at it. Once the bird completed all trials within a session, it was presented with a modified training procedure. The images were the same as those used in training except that a black square was present in the correct corner only. Preliminary training continued until a bird completed at least 80 (out of a total of 96) trials on three consecutive sessions.

Feature training. Six images of a schematic rectangular environment were presented in a randomly determined order across trials. In each image, a distinctive feature was located adjacent to each corner of the rectangular environment. (Each feature had a unique color and shape.) The position of the correct corner was counterbalanced across subjects. Pecks to the correct black square were immediately reinforced; pecks directed at an incorrect square removed the image display, turned off the house light, and instituted a 2-s ITI. Once the bird obtained $80 \%$ or greater accuracy on three consecutive sessions and completed a minimum of 80 trials per session, the percentage of trials on which reinforcement was available was reduced from $100 \%$ to $75 \%$ and then to $50 \%$. On nonreinforced trials, pecks to a black square terminated the display, but food was never presented. We adopted this procedure to familiarize the birds with the absence of reinforcement. Training with $50 \%$ reinforcement continued until a pigeon completed at least 80 (of a total of 96 ) trials with $80 \%$ or greater accuracy on three consecutive sessions, and then the pigeons were moved onto testing.

Feature testing. Several testing conditions were presented in a counterbalanced order across subjects (with the exception of the identical and all correct tests, the order of which are described below). Three trial types were presented during each testing condition: baseline trials, control trials, and test trials. All baseline trials presented the same images as those used in training, and if the pigeons responded correctly, they received reinforcement. Control trials again presented the same images as in training, but responses were never reinforced. Test trials manipulated some aspect of the image and were always presented without reinforcement. Each testing session consisted of 96 trials: 48 baseline trials ( 8 at each of the 6

rotations), 24 control trials (4 at each rotation), and 24 test trials (4 at each rotation). The order of the trials was random. Each testing condition comprised a minimum of eight testing sessions.

The testing conditions examined (a) the influence of the appearance or location of the rectangular environment (no shading test and move test), (b) the influence of the distinct features (color-only test, shape-only test, and distant test), and (c) whether geometry was encoded (identical tests and all correct tests) and whether the representation withstood rotations (new rotation test).

For the no shading test, the gray shaded rectangle was removed, but the black squares and the distinct features at the corners remained identical to the training trials (see Figure 1B). This manipulation assessed whether the continuous gray surface of the rectangle was an important aspect of the environment. The move test presented the same images as in training but displayed them in the upper left or lower right portions of the display, rather than in the center. This manipulation examined whether featural and geometric representations would withstand spatial translations.

The color-only test presented the same rectangular environment as in training, but the four features were all presented in the shape of a formée

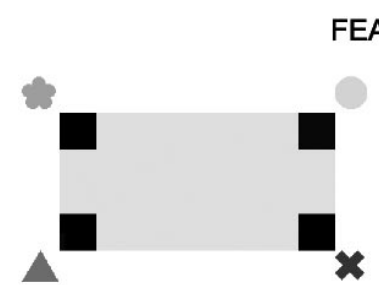

A) FEATURE CONTROL

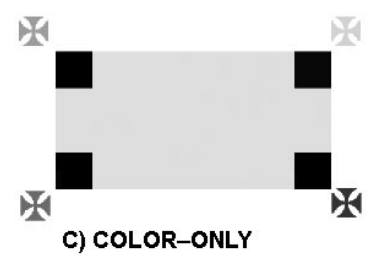

C) COLOR-ONLY

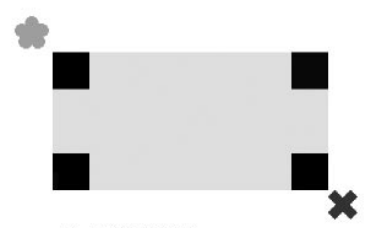

E) DISTANT

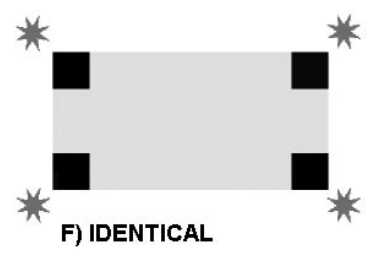

Figure 1. Examples of the images used in feature testing. A: Feature control. B: No shading test. C: Color-only test. D: Shape-only test. E: Distant test. F: Identical test. G: All correct test. For the purposes of illustration, all of these examples are drawn as if the triangle (presented as red on the monitor) was the feature in the correct corner, although this was counterbalanced across subjects. The move test is not shown in this figure. 
cross (see Figure 1C). The four identically shaped features were presented in the same distinct color of the features during training. This test examined whether the pigeons encoded the color of features. In the shape-only test, the distinct colors were removed and all features were presented in an identical purple color, but the unique shapes of the features remained the same as in training (see Figure 1D). Therefore, this test examined whether the pigeons encoded the unique shapes of the features. The distant test examined whether pigeons encoded features at the corners distant to the correct one (see Figure 1E). For this test, the features in the correct corner and the diagonally opposite corner were removed. Therefore, to respond correctly during this test, the pigeons had to have encoded something about the features in one, or both, of the distant corners.

The identical and all correct tests were conducted to assess encoding of geometry, and they provided two different ways of removing the distinctive featural information. During the identical tests, the four distinctive features were replaced by four identical eight-sided brown stars (the color and shape were both different from any training feature; see Figure 1F), and during the all correct tests, the distinctive features were replaced by the feature in each bird's correct corner. (An example of the all correct test for the pigeon with the red triangle as correct is shown in Figure 1G.) These testing conditions were presented in two phases: one phase before all other feature tests, and the second phase following the completion of the other feature tests, so that we could determine whether accuracy in using the geometry changed after the pigeons had experience with the feature tests. These tests are referred to as identical-before, identical-after, all correctbefore, and all correct-after, respectively.

Geometric training. The training procedures were very similar to the feature training, so only the differences are explained here. Pigeons were presented with the same six images depicting the rectangular environment; however, unlike in featural training, none of these images contained distinctive features. The only source of information the birds had to distinguish the corners was the geometric information supplied by the shape of the environment itself. However, because of the symmetry of the rectangular environment, it is impossible to distinguish the correct corner from the corner diagonally opposite to it (the geometrically equivalent corner). Therefore, although only one corner was "correct" (i.e., provided reinforcement), in our calculation of accuracy we counted responses to the correct corner and the geometrically equivalent corner as correct responses.

Geometric testing. The testing procedures were similar to those used in featural testing except that none of the distinctive features were present (see Figure 2A). The four testing conditions were the move test, no shading test, new rotation test, and identical test (see Figures 2B, 2C, and 2D for each of these respective tests).

The move test examined whether the pigeons' encoding of geometry would withstand spatial translations, and it displayed the rectangular environment in either the lower right or the upper left corner of the screen, rather than in the center of the monitor. For the no shading test, the shaded rectangle was removed to determine whether the continuous gray surface of the rectangle was important in encoding the geometry of the environment (see Figure 2B). The new rotation test presented the rectangular environment in the two novel rotations that the birds had not experienced during training (see Figure 2C). For instance, the new rotation test for subgroup $0^{\circ}-180^{\circ}$ showed the geometric environment at the two rotations $0^{\circ}$ and $180^{\circ}$. The identical test was presented in two phases, as the first geometric test and as the last geometric test. This was done, as in featural testing, to evaluate whether control by geometry changed over the course of testing.

\section{Data Analysis}

Only data from the nonreinforced control and test trials were analyzed. We calculated the percentage of choices (\% choice) made to a particular corner averaged over all pigeons in the particular group. Our criterion for significance was $p<.05$ for all statistical tests unless otherwise stated.

\section{GEOMETRIC TESTS}

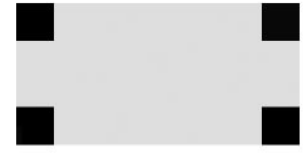

A) GEOMETRIC CONTROL

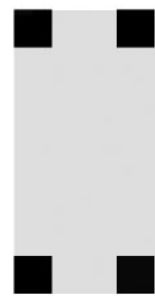

C) NEW ROTATION

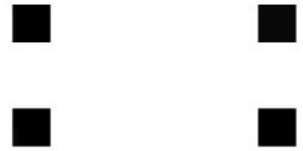

B) NO SHADING

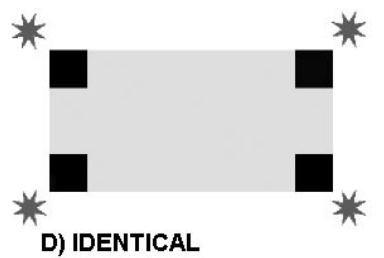

Figure 2. Examples of the images used in geometric testing. A: Geometric control. B: No shading test. C: New rotation test. D: Identical test. The correct corner was counterbalanced across subjects. The move test is not shown in this figure.

Initial data analyses were conducted using an analysis of variance (ANOVA) for mixed-factor designs, followed by Fisher's least significant difference (LSD) tests when a significant $F$ ratio was found. Additional $t$ tests were conducted to test specific hypotheses about choices to one or two corners. For testing conditions in which different kinds of tests were presented on separate sessions, we conducted Fisher's LSD tests to determine whether accuracy on control trials differed across sessions. None of these analyses revealed any significant differences, and so data from the control trials were pooled for comparison with the tests.

\section{Results}

Group F-G required fewer sessions $(M=27.5)$ to learn the initial task (feature training) than did group G-F (geometric training; $M=45.75)$ : $t(6)=-2.18, p<.05$, one-tailed. Relearning of the second task also progressed at dramatically different rates for the two groups: Group F-G learned the second task (geometric training) in a mean of 7.5 sessions, whereas group G-F required a mean of 23.5 sessions to learn the second task (featural training); this difference was significant: $t(6)=-3.53, p<.01$, one-tailed.

\section{Featural Testing}

Group F-G. We first examined the pigeons' responses to manipulations of the appearance and location of the rectangular environment (i.e., no shading and move tests). The two move tests (63.7\% and $63.8 \%$ for the lower right and upper left move tests, respectively) did not differ from each other, $t(3)=-0.05, p>.1$, so these two test types were pooled in the following analyses. A repeated measures ANOVA showed that group F-G responded differently to these two types of manipulations: trial type, $F(2$, $6)=31.09, p<.001$. The feature control tests $(91.5 \%)$ were significantly different from the move tests $(63.7 \%)$ but were not different from the no shading test $(94.3 \%$; see Figure $3 \mathrm{~A})$. The no shading test was also significantly different from the move tests. A 

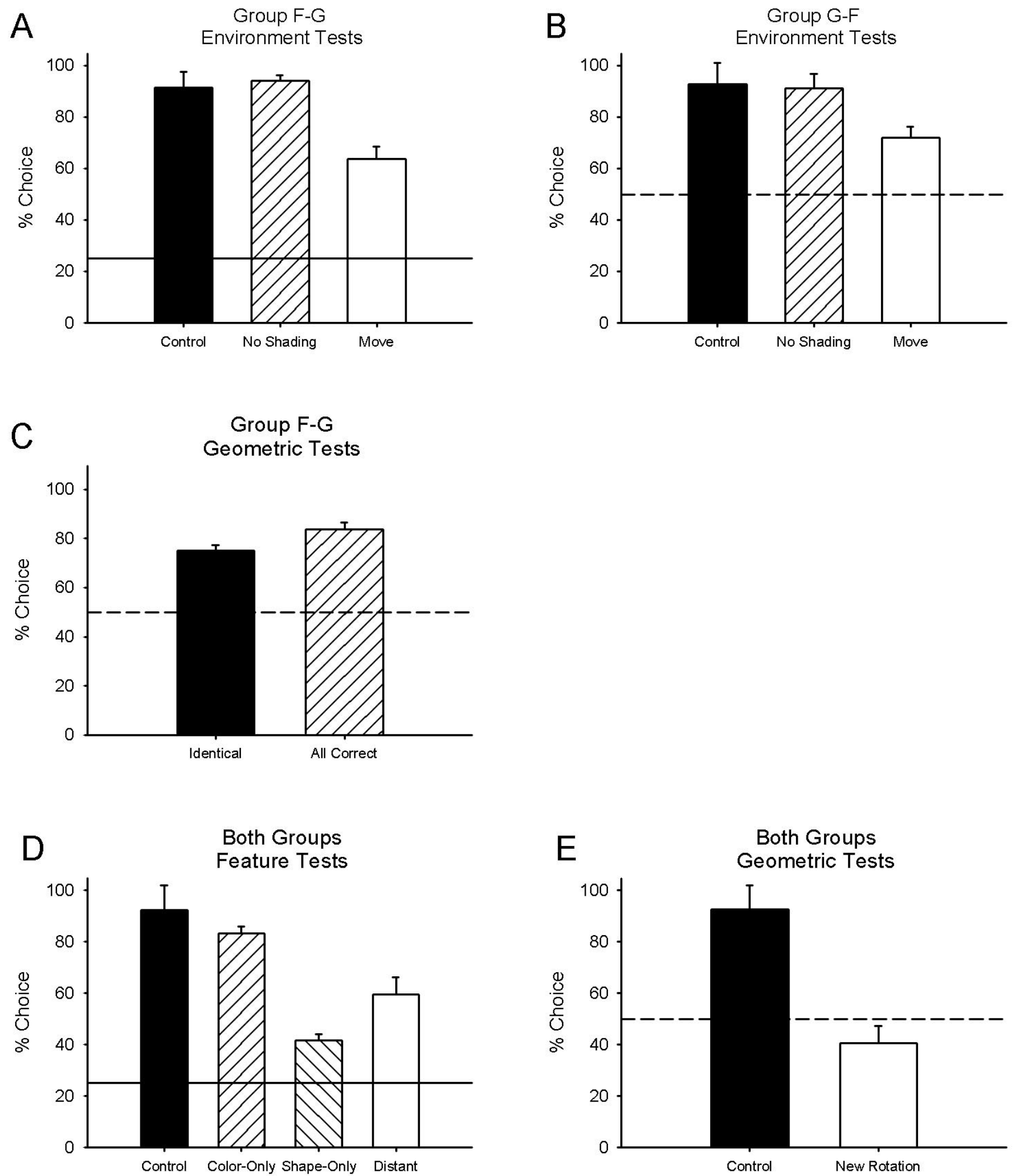

Figure 3. A: Percentages of choices to the correct corner by group F-G for feature control and the no shading and move tests. B: Percentages of choices to the two geometrically correct corners by group G-F for the geometric control and the no shading and move tests. C: Percentages of choices to the two geometrically correct corners by group F-G for the average of the two identical tests and the two all correct tests. D: Percentages of choices to the correct corner averaged for both groups F-G and G-F for the feature tests: feature control, color-only, shape-only, and distant tests. E: Percentages of choices to the two geometrically correct corners averaged for both groups F-G and G-F for the geometric control and new rotation test. Error bars represent the standard errors of the mean. The solid lines indicate chance levels for encoding featural information (25\%); the dashed lines indicate chance levels for encoding geometric information $(50 \%) \mathrm{F}=$ feature; $\mathrm{G}=$ geometry. 
one-sample $t$ test of the pooled data showed that although the move tests were significantly different from the feature control tests, the birds still chose the featurally correct corner more often than expected by chance $(50 \%): t(7)=2.90, p<.05$. Thus, the pigeons' accuracy in choosing the positive corner was not disrupted by removal of the gray shading. When the entire rectangular environment was shifted from the center of the screen to either the upper left or the lower right corner, accuracy dropped significantly from the feature control tests, but the birds continued to choose the positive corner more often than expected by chance.

Did group F-G encode the geometric properties of the rectangle (or the configuration of the four black squares) even though this was not necessary to learn the task? The geometry test allowed us to examine this possibility. If the pigeons encoded the geometric properties, we would expect that when we removed all the featural cues the birds would respond primarily to the two geometrically correct corners. To determine whether this was the case, we examined the percentage of total choices made to the two geometrically correct corners on the identical tests and the all correct tests. A repeated measures ANOVA showed that the two test types were significantly different from each other: trial type, $F(3,9)=7.46$, $p<.01$. However, a Fisher's LSD test revealed no significant differences between the before and after tests, respectively, for either the identical tests $(73.9 \%$ and $76.2 \%)$ or the all correct tests $(82.3 \%$ and $85.3 \%)$, and therefore the before and after tests were pooled for further analyses. A one-sample $t$ test showed that with both the identical tests $(75.0 \%)$ and the all correct tests $(83.8 \%)$, the pigeons chose the geometrically correct corners more often than expected by chance (50\%): $t(7)=11.35$, and $t(7)=12.28$, respectively, all $p \mathrm{~s}<.01$ (see Figure $3 \mathrm{C}$ ). Furthermore, paired $t$ tests showed no differences between the positive corner and the geometrically equivalent corner for the identical tests $(37.9 \%$ and $37.1 \%$, respectively) and the all correct tests $(45.5 \%$ and $38.3 \%$, respectively): $t(3)=1.15$, and $t(3)=3.33, p \mathrm{~s}>.05$, respectively. This result shows that the pigeons in group F-G spontaneously encoded the geometric properties of the environment even though such encoding was not required. The fact that the birds in group F-G spontaneously encoded the geometric information perhaps clarifies why group F-G required so few sessions to be retrained with geometry.

Both groups. Next, we examined the tests that manipulated the distinctive features (color-only, shape-only, and distant tests). We considered choices to the corner containing the feature with the correct color (color-only tests), the correct shape (shape-only tests), or the corner where the correct feature would be (distant tests) as a correct choice. A mixed-factor ANOVA, Group (F-G and G-F) $\times$ Trial Type (feature control tests, color-only tests, shape-only tests, and distant tests), showed no effect of group: $F(1$, $6)=2.03, p>.05$. However, a significant effect of trial type was found: $F(3,18)=33.76, p<.0001$. The feature control tests $(92.2 \%)$ were significantly different from both the shape-only $(41.6 \%)$ and distant $(59.6 \%)$ tests, but were not different from the color-only test $(83.3 \%$; see Figure $3 \mathrm{D})$. The color-only test was significantly different from both the shape-only test and the distant test. These latter two tests were not significantly different from each other. One-sample $t$ tests showed that the pigeons did not choose the correct corner on the distant test and were below geometric chance on the shape-only test: $t(7)=1.44$, and $t(7)=$ -3.28 , respectively. However, they chose the correct corner more often than the diagonally opposite corner on both the shape test$t(7)=3.44, p<.05$ - and the distant test-t(7) $=3.63, p<$ .01 -indicating that they nevertheless were able to use both the shape and the distant cues to differentiate between the two geometrically correct corners.

\section{Geometric Testing}

Group $G-F$. Again, we first examined the pigeons' responses to manipulations of the overall environmental information (i.e., no shading and move tests). The two move tests were not significantly different from each other $(79.5 \%$ and $64.3 \%$ for the lower right and upper left move tests, respectively), $t(3)=2.10, p>.05-$ so the tests were pooled in further analyses. A repeated measures ANOVA revealed a significant effect of trial type: $F(2,6)=29.66$, $p<.001$. The geometric control tests $(92.8 \%)$ were significantly different from the move tests $(71.9 \%)$, but were not different from the no shading test $(91.1 \%$; see Figure 3B). A Fisher's LSD test revealed that the no shading test was significantly different from the move tests $(p<.05)$. A one-sample $t$ test showed that although accuracy on the move tests was significantly lower than on the geometric control tests, the birds nevertheless chose the geometrically correct corners more often than expected by geometric chance (50\%): $t(7)=5.15, p<.01$. Finally, paired $t$ tests showed that the pigeons distributed their choices equally between the two geometrically correct corners on the no shading tests and the move tests: $t(3)=-0.28$, and $t(7)=1.70$, respectively, both $p \mathrm{~s}>.1$.

In summary, the results from the no shading test showed that the pigeons could use the geometric properties defined by the configuration of the four black squares; thus, geometry did not have to be defined by a continuous surface. In addition, the geometric representation survived translations: Although accuracy dropped from the geometric control tests, the pigeons continued to choose the correct corners more often than expected by chance when the entire rectangular environment was shifted from the center of the monitor's screen to either the upper left or the lower right corner.

Both groups. To examine the flexibility of the geometric encoding, we manipulated the orientation of the rectangular environment by presenting the environment in a novel orientation. A mixed-factor ANOVA, Group (F-G and G-F) $\times$ Trial Type (geometric control and new rotation tests), showed no effect of group: $F(1,6)=1.18, p>.05$. However, a significant effect of trial type was found: $F(1,6)=46.93, p<.001$. This shows that the average percentage of choices to the two geometrically correct corners on the geometric control test $(92.7 \%)$ was significantly different from the choices to the two geometrically correct corners on the new rotation test (40.6\%; see Figure 3E). A one-sample $t$ test showed that on the new rotation test, the pigeons did not respond to the two geometrically correct corners more often than expected by chance (50\%): $t(7)=-1.40, p>.1$.

To examine whether the pigeons became more accurate in their choices to geometry over the course of the experiment, we conducted an identical-before and an identical-after test. A mixedfactor ANOVA, Group (F-G and G-F) $\times$ Trial Type (identicalbefore and identical-after tests), showed no effect of group: $F(1$, $6)=0.19, p>.1$. However, a significant effect of trial type was found: $F(1,6)=6.53, p<.05$. The average percentage of choices to the two geometrically correct corners was significantly lower on the identical-before test $(86.6 \%)$ than on the identical-after test 
(93.5\%), indicating that the birds' accuracy in choosing the two geometrically correct corners increased over the course of testing. One-sample $t$ tests showed that the pigeons continued to choose between the two geometrically correct corners equally, $t(23)=$ -0.23 , and $t(23)=-1.99$, respectively, both $p \mathrm{~s}>.05$; before and after tests were pooled.

\section{Experiment 1B}

In Experiment $1 \mathrm{~B}$ we further examined the extent to which pigeons encoded the individual features. Experiment $1 \mathrm{~A}$ revealed that the pigeons could use the features in the geometrically incorrect corners to choose the correct corner over the diagonally opposite corner. In this experiment, we included tests to assess whether both or only one of the distant features were encoded. Specifically, we removed the features in both of the geometrically correct corners and in one of the geometrically incorrect corners, leaving only the feature in the corner along the short wall or in the corner along the long wall from the correct corner.

We also conducted conflict tests to determine the relative weighting of featural and geometric cues. To pit the two types of information against each other, we used an affine transformation, which moves each feature one position either clockwise or counterclockwise while maintaining the order of the featural cues. Thus, the features that were in geometrically correct locations during training were placed in geometrically incorrect corners during testing. The pigeons could use only the featural cues, only the geometric cues, or some combination of both cues when the features and geometric information were in conflict. In a navigable environment, we found that featural cues dominated when pigeons were trained with both geometry and features from the outset, but both cues were used when pigeons were trained with geometry alone before being trained with both features and geometry (Kelly et al., 1998).

\section{Method}

\section{Subjects}

The pigeons from groups F-G and G-F from Experiment 1A were used in this experiment directly after completion of Experiment $1 \mathrm{~A}$.

\section{Apparatus and Images}

The apparatus and training images were identical to those used in feature training of Experiment $1 \mathrm{~A}$

\section{Training and Testing Procedures}

Feature training. After completion of all training and testing conditions in Experiment 1A (including the retraining procedures), all birds from both groups received featural training (as described in Experiment 1A) for a minimum of 3 days. Once the birds maintained $80 \%$ accuracy on 3 consecutive days, the testing procedures were started.

Feature testing. Many of the testing procedures and number of trials were identical to the feature testing in Experiment 1A; therefore, only the exceptions are described here. Two types of testing conditions were examined: (a) whether both distant features were encoded (distant-near and distant-far tests) and (b) the use of geometric and featural information when these sources of information provided conflicting information (affine-clockwise and affine-counterclockwise tests). The test types were presented sequentially; however, the order of testing was counterbalanced across subjects (i.e., half the birds received distant testing followed by affine testing and vice versa).

For the distant-near test, the featural information was removed from the two geometrically correct corners and from the corner farthest from the correct one. This left only the feature in the corner nearest to the correct corner along the short wall (see Figure 4A). For the distant-far test, the features in both geometrically correct corners and in the corner nearest to the correct corner were removed, leaving only the feature in the incorrect corner along the long wall from the correct corner (see Figure 4B). The affine-clockwise and affine-counterclockwise tests pitted featural information against geometric information by moving each feature either one corner clockwise or counterclockwise, respectively (see Figures 4C and 4D).

\section{Results}

For the distant tests, choices to the corner that would have contained the correct feature, based on the training configuration of landmarks, were scored as correct. A mixed-factor ANOVA, Group $(\mathrm{F}-\mathrm{G}$ and G-F) $\times$ Test Type (feature control, distant-near, and distant-far tests), showed no effect of group: $F(1,6)=3.17$, $p>$.1. However, a significant effect of test type was found: $F(1$, $12)=56.69, p<.0001$. The feature control tests $(94.7 \%)$ were significantly different from both the distant-near (54.9\%) and the distant-far $(44.0 \%)$ tests, which were also significantly different from each other (see Figure 5A). One-sample $t$ tests showed that the pigeons did not choose the correct corner on either of these tests more often than expected by geometric chance (50\%): $t(7)=$ 0.93 , and $t(7)=-1.86$, respectively, all $p \mathrm{~s}>0.1$. However, the pigeons chose the correct corner more often than the diagonally opposite corner on the distant-near test (54.9\% vs. $30.5 \%): t(7)=$ $2.72, p<.05$; and on the distant-far test $(44.0 \%$ vs. $36.7 \%): t(7)=$ $2.44, p<.05$.

For the tests pitting features against geometry, we scored choices to the corner containing the correct feature as correct. Initial testing indicated no differences between the two affine transformations $(31.1 \%$ and $33.5 \%$, respectively), $t(7)=-1.19$, $p>.1$, and therefore these tests were pooled for further analyses.
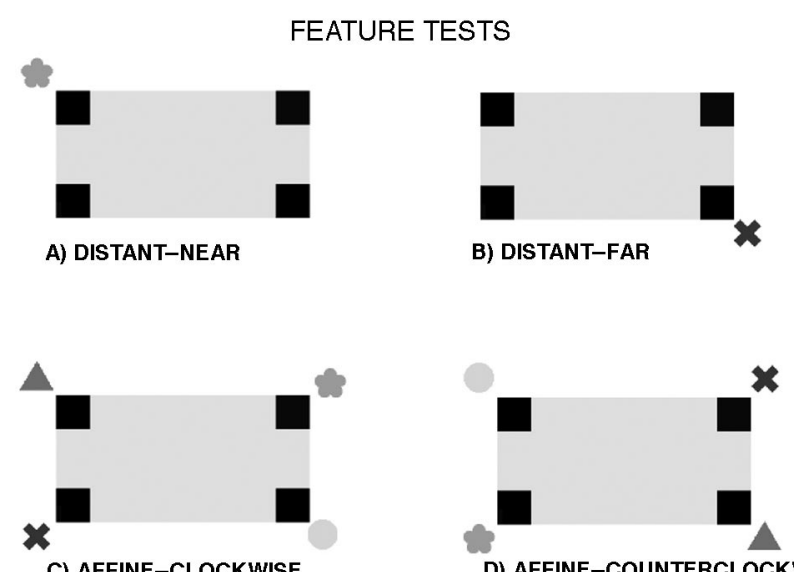

D) AFFINE-COUNTERCLOCKWISE

Figure 4. Examples of the images used in feature testing. A: Distant-near test. B: Distant-far test. C: Affine-clockwise test. D: Affine-counterclockwise test. For the purposes of illustration, all of these examples are drawn as if the lower left corner was the correct corner, although this was counterbalanced across subjects. 

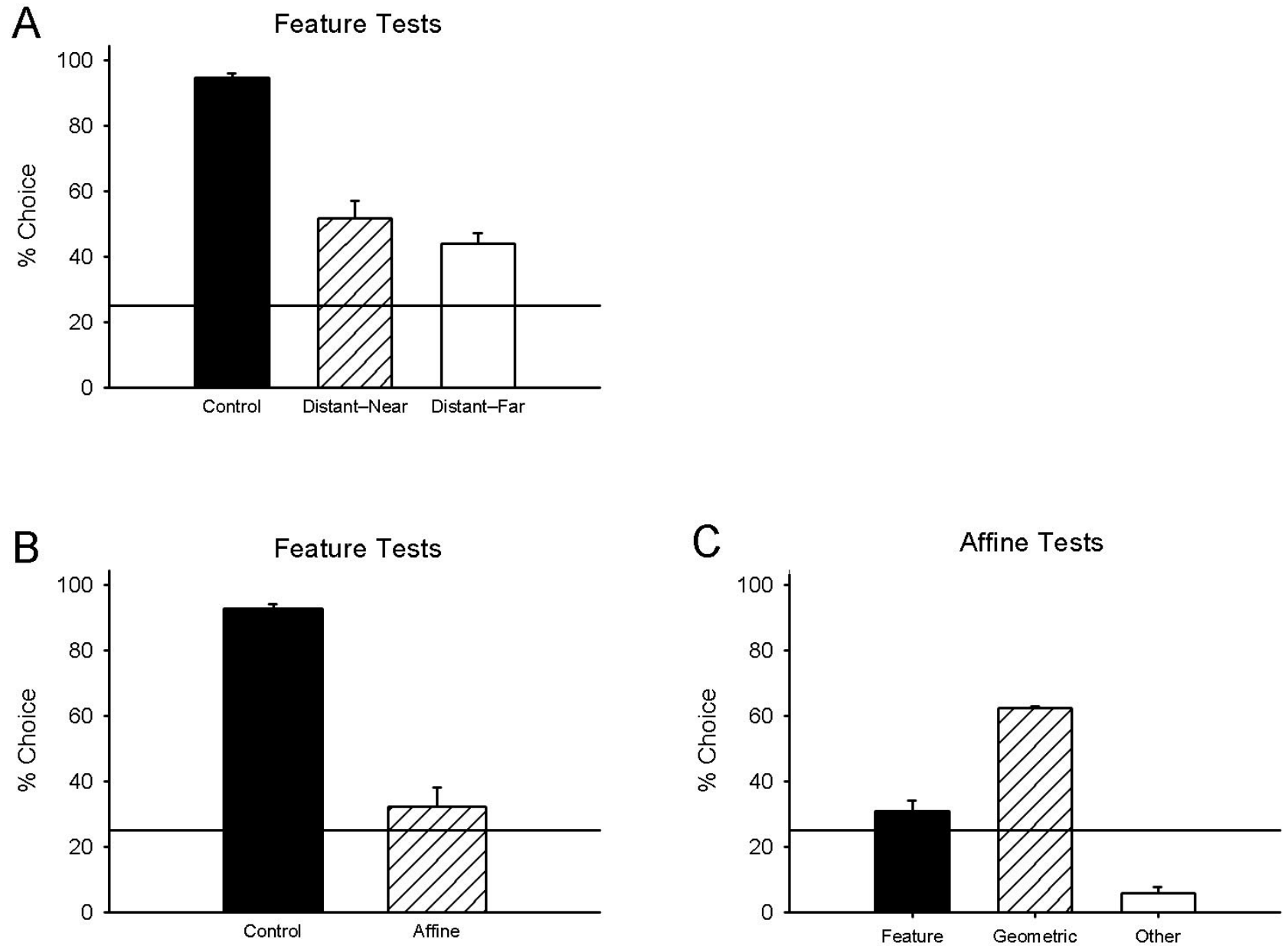

Figure 5. A: Percentages of choices to the correct corner for the feature control and the distant-near and distant-far tests. B: Percentages of choices to the correct corner averaged for the feature control and the affine tests. C: Percentages of choices to the featurally correct corner, the two geometrically correct corners, and the remaining corner averaged across both affine tests. Error bars represent standard errors of the mean. The solid lines indicate chance levels for encoding featural information.

A mixed-factor ANOVA, Group $(\mathrm{F}-\mathrm{G}$ and G-F) $\times$ Trial Type (feature control tests and affine tests), showed no effect of group: $F(1,6)=0.02, p>$.1. However, a significant effect of trial type was found: $F(1,6)=53.96, p<.001$. The feature control tests $(93.0 \%)$ were significantly different from the affine tests $(32.3 \%)$. A one-sample $t$ test showed that the pigeons did not choose the correct corner on the affine tests more often than expected by chance $(25 \%): t(15)=1.26, p>.1$ (see Figure 5B). However, the pigeons did choose the featurally correct corner more often than the diagonally opposite corner (5.8\%): paired $t$ test, $t(15)=4.47$, $p<.001$.

Next, we examined choices to the two geometrically correct corners for the two test types. A one-sample $t$ test showed that the pigeons chose the two geometrically correct corners $(62.3 \%)$ more than expected by chance (50\%): $t(15)=2.15, p<.05$ (see Figure $5 \mathrm{C})$. Furthermore, the birds divided their choices equally between the two geometrically correct corners $(31.3 \%$ and $31.0 \%$, respectively): $t(15)=0.06, p>.1$.

\section{General Discussion}

Our results show that pigeons can conjoin featural and geometric information when these properties are presented with a schematic of a rectangular environment, a result we also found in an earlier study with a 3-D navigable environment (Kelly et al., 1998). Across the two experiments, we found several notable results regarding the way in which pigeons encoded geometry and features in our 2-D space.

\section{Encoding of Geometry}

Our results clearly showed that pigeons encoded the geometric properties of the schematic environment. They not only learned to use geometric cues when they were trained without features, but birds that were trained initially with distinctive features showed spontaneous encoding of the geometric information even though this was not necessary to solve the task. In addition, pigeons were 
able to use the geometric information supplied by the configuration of the discrete four black squares when the continuous gray shading was removed. These results are consistent with those from humans in our companion study (Kelly \& Spetch, 2004) and are very interesting in light of evidence that young children can reorient according to the geometry of surfaces but not of discrete landmarks in a navigable environment (Gouteaux \& Spelke, 2001; but see Garrad-Cole, Lew, Bremner, \& Whitaker, 2001). The encoded geometric representation was also flexible in that it was not tied to a specific screen location; pigeons were somewhat disrupted but nevertheless continued to show significant control by geometry when the environment was translated above or below its training position on the screen. Interestingly, however, the representation was not independent of orientation: When we presented the environment in a novel orientation, the pigeons did not respond to the geometrically correct corners.

Although geometry was learned and spontaneously encoded, initial acquisition of the task was slower for birds trained with geometry alone than for birds trained with features. This result parallels our findings from a similar experiment with humans (Kelly \& Spetch, 2004). In that study, significantly more participants learned the task within the allotted training trials in the feature condition than in the geometry-only condition. The slower initial acquisition in the geometry condition, coupled with the finding that control by geometry did not generalize to novel rotations, suggests the possibility that the pigeons may have used a multiple orientation-specific coding strategy. Specifically, whereas acquisition in the feature condition may have required the encoding of only a single feature map, acquisition in the geometryonly condition may have involved the learning of unique codes for each distinctive orientation of the environment in the geometric condition. As suggested in our companion study (Kelly \& Spetch, 2004), the use of a vertical-orientation search space on the computer screen may have provided a gravity-defined privileged axis, which may have encouraged orientation-specific encoding. Furthermore, in contrast to active navigation through a 3-D environment, participants in the current task were passive to the orientation changes of the environment. Additional research manipulating the nature of the environment, the orientation changes, and the viewer is needed to determine the conditions that contribute to orientation-specific encoding of geometry (see Kelly \& Spetch, 2004, for further discussion).

\section{Encoding of Features}

The pigeons readily learned to use features to choose the correct corner. Moreover, as found in previous studies with pigeons in a 3-D navigable environment, the pigeons' encoding of features was not restricted to the features found in the geometrically correct corners. When we removed both features in the geometrically correct corners (distant test in Experiment 1A and near and far tests in Experiment 1B), the pigeons still chose the correct corner significantly more often than expected by chance. The use of distant features by pigeons in both the 2-D and 3-D environments is a very interesting result because several other species (rats: Cheng, 1986; chicks: Vallortigara et al., 1990; fish: Sovrano et al., 2003) have failed to show learning of the features in the geometrically incorrect corners. However, we found encoding of distant features by humans in our companion study with the 2-D sche- matic (Kelly \& Spetch, 2004). Thus, whereas the rats, chicks, and fish may have encoded only the feature nearest the goal for use as a beacon, the pigeons and humans encoded a broader feature map and appeared to use the features as landmarks.

Although our results suggest that the pigeons encoded a broad feature map, our results also indicate that the featural information was not all weighted equally. First, removal of the distinctive shape information from the features had no significant effect on choice behavior, whereas removal of the distinctive color information significantly disrupted accuracy. The birds, however, still accurately distinguished between the two geometrically correct corners when color was removed. Together, these results suggest that the color of the correct feature was relied on more strongly than shape information, yet the shape information was encoded sufficiently to allow for discrimination. Again, this result is very similar to that found for pigeons in a navigable environment (Kelly et al., 1998). In our companion study (Kelly \& Spetch, 2004), humans also showed encoding of both the shape and color of the features, but unlike the pigeons, color information was not relied on more strongly than shape information. Second, the pigeons showed stronger control by the feature at the geometrically incorrect corner that was closest to the goal than by the feature at the farthest geometrically incorrect corner. This greater weighting of landmarks closer to a goal has been found in numerous studies of landmark-based searching (see Cheng \& Spetch, 1998).

\section{Relationship Between the Encoding of Geometry and Features}

Although it is very interesting that pigeons' pattern of cue use in this 2-D environment is similar to that reported in a 3-D navigable environment, a perhaps more interesting question is how does the use of featural and geometric information interact? We examined this question in two ways. First, we examined whether training with featural information competed with the encoding of geometric cues. We found that pigeons trained with features showed systematic rotational errors when the features were removed, indicating that they had indeed encoded geometry, even though this was not required to solve the task. This result replicates that found in the open-field setting (Kelly et al., 1998) and is very interesting because it shows that even in a very spatially limited schematic of an environment, pigeons still spontaneously encode geometry. Moreover, the prior training with features did not interfere with subsequent acquisition of geometry learning for group F-G. Instead, the birds in group F-G learned the geometry condition exceedingly fast (7.5 sessions compared with 45.0 sessions for group G-F), likely because they had already spontaneously encoded geometry. This contrasts with the similar learning of features for the two groups (27.5 and 23.5 sessions for groups F-G and G-F, respectively). If anything, it appears that learning of the features may facilitate the learning of geometry, an effect that is opposite to cue competition. In contrast to the cue competition that often occurs between landmarks (Cheng \& Spetch, 2001; Roberts \& Pearce, 1999; Rodrigo, Chamizo, McLaren, \& Mackintosh, 1997; Spetch, 1995), other studies have also found little or no evidence for competition between beacons and geometry (Hayward, McGregor, Good, \& Pearce, 2003; Pearce, Ward-Robinson, Good, Fussell, \& Aydin, 2001), and one study found that beacons sometimes facilitate the learning of geometry (Pearce et al., 2001). 
Another finding in the present study that is consistent with the notion that features may facilitate control by geometry comes from the comparison of the geometry tests in which the distinctive features were replaced with identical novel features or identical versions of the correct feature: Accuracy was significantly higher when the correct feature was present in each corner even though it was not informative about the location of the correct corner.

Second, given that pigeons encoded both geometry and features, we examined which cue type would guide the pigeons' choices if the two sources of information gave conflicting information. On affine transformation tests, which pitted featural and geometric information against each other, pigeons in both groups divided their choices between the featurally correct corner and the two geometrically correct corners. This result is similar to that reported in an open-field setting for birds initially trained to use only geometric information (Kelly et al., 1998). However, in this earlier research, birds that were trained from the outset with distinct featural information in each corner of a rectangular enclosure showed strong control by features on an affine transformation. In contrast to these results, we did not find a difference between birds initially trained with features (group F-G) and birds trained first with geometry alone (group G-F). Instead, both groups divided their choices among the two geometrically correct corners and the featurally correct corner almost perfectly equally $(31.2 \%, 31.1 \%$, and $32.3 \%$, respectively). One potentially important difference between the two studies is that birds trained initially with features in the present study received subsequent training with geometry only, whereas such training was not provided for birds in the feature group of Kelly et al. (1998). Thus, training with geometry alone, either before or after training with features and geometry, may be sufficient to produce equal weighting of geometry and features. However, the equal weighting of geometry and features after training with both may be species specific. Human participants who were trained with the same 2-D environment as used here were found to rely almost exclusively on featural information on affine tests; they shifted their responses to the geometrically incorrect corner that contained the correct featural cue (Kelly \& Spetch, 2004). Unfortunately, to our knowledge, no studies have tested adult humans with affine transformations in a 3-D navigable environment.

In summary, the present findings significantly further our understanding of the use of featural and geometric information for orientation in a 2-D environment. In our companion study (Kelly \& Spetch, 2004), we found that human adults showed conjoining of geometric and featural information in the same 2-D environment used here. The adults showed strong control by featural cues but also showed spontaneous encoding of geometry. In that study, we contemplated how these results with human participants would generalize to other species, given that humans have extensive experience interpreting maplike representations of real environments, whereas animals do not. The results from this present study show that the results from our human participants generalize well to pigeon subjects. Our pigeons also conjoined featural and geometric information, and they learned and spontaneously encoded geometric information. Furthermore, the way geometric information was encoded was remarkably similar between the two species. For both humans and pigeons, the encoded geometry withstood translations and removal of the shading but did not withstand novel orientations. One species difference emerged when geometry and features provided conflicting information: Humans showed strong reliance on featural information, whereas pigeons divided their choices equally between the location defined by the correct feature and the locations defined by the correct geometry.

The current study and our companion study with humans (Kelly $\&$ Spetch, 2004) have allowed us to examine how organisms use featural and geometric information when orienting in a 2-D schematic environment. Yet many interesting questions remain. For instance, pigeons trained and tested in 3-D environments use both relative and absolute metrics when encoding surface geometry (Gray, Spetch, Kelly, \& Nguyen, 2004; Kelly \& Spetch, 2001). Would pigeons show similar encoding in a $2-\mathrm{D}$ environment? Is orientation-specific encoding of geometry on the computer-screen task general across species? In particular, would rats and young children, which both have been found to show very strong and dominant control by geometry in 3-D tasks, also show orientation specificity in vertically oriented 2-D tasks? Addressing these and other questions will add to our growing knowledge about the various spatial orientation mechanisms used by different species in various environments.

\section{References}

Cheng, K. (1986). A purely geometric module in the rat's spatial representation. Cognition, 23, 149-178.

Cheng, K., \& Gallistel, C. R. (1984). Testing the geometric power of an animal's spatial representation. In H. L. Roitblat, T. G. Bever, \& H. S. Terrace (Eds.), Animal Cognition (pp. 409-423). Hillsdale, NJ: Erlbaum.

Cheng, K., \& Spetch, M. L. (1998). Mechanisms of landmark use in mammals and birds. In S. Healy (Ed.), Spatial representation in animals (pp. 1-17). Oxford, England: Oxford University Press.

Cheng, K., \& Spetch, M. L. (2001). Blocking in landmark-based search in honeybees. Animal Learning \& Behavior, 29, 1-9.

Gallistel, C. R. (1990). The organization of learning. Cambridge, MA: MIT Press.

Garrad-Cole, F., Lew, A. R., Bremner, J. G., \& Whitaker, C. J. (2001). Use of cue configuration geometry for spatial orientation in human infants (Homo sapiens). Journal of Comparative Psychology, 115, 317-320.

Gouteux, S., \& Spelke, E. S. (2001). Children's use of geometry and landmarks to reorient in an open space. Cognition, 81, 119-148.

Gouteux, S., Thinus-Blanc, C., \& Vauclair, J. (2001). Rhesus monkeys use geometric and non-geometric information during a reorientation task. Journal of Experimental Psychology: General, 130, 505-519.

Gouteux, S., Vauclair, J., \& Thinus-Blanc, C. (2001). Reorientation in a small-scale environment by 3-, 4-, and 5-year-old children. Cognitive Development, 16, 853-869.

Gray, E., Spetch, M. L., Kelly, D. M., \& Nguyen, A. (2004). Searching in the center: Pigeons encode relative distances from walls of an enclosure. Journal of Comparative Psychology, 118, 113-117.

Hayward, A., McGregor, A., Good, M. A., \& Pearce, J. M. (2003). Absence of overshadowing and blocking between landmarks and the geometric cues provided by the shape of a test arena. Quarterly Journal of Experimental Psychology, 56B, 114-126.

Healy, S. A. (Ed.). (1998). Spatial representation in animals. Oxford, England: Oxford University Press.

Hermer, L., \& Spelke, E. S. (1994, July 7). A geometric process for spatial reorientation in young children. Nature, 370, 57-59.

Hermer, L., \& Spelke, E. S. (1996). Modularity and development: The case of spatial reorientation. Cognition, 61, 195-232.

Kelly, D. M., \& Spetch, M. L. (2001). Pigeons encode relative geometry. Journal of Experimental Psychology: Animal Behavior Processes, 27, 417-422. 
Kelly, D. M., \& Spetch, M. L. (2004). Reorientation in a two-dimensional environment: I. Do adults encode the featural and geometric properties of a two-dimensional schematic of a room? Journal of Comparative Psychology, 118, 82-94.

Kelly, D. M., Spetch, M. L., \& Heth, C. D. (1998). Pigeons' (Columba livia) encoding of geometric and featural properties of a spatial environment. Journal of Comparative Psychology, 112, 259-269.

Margules, J., \& Gallistel, C. R. (1988). Heading in the rat: Determination by environmental shape. Animal Learning \& Behavior, 16, 404-410.

Pearce, J. M., Ward-Robinson, J., Good, M., Fussell, C., \& Aydin, A. (2001). Influence of a beacon on spatial learning based on the shape of the test environment. Journal of Experimental Psychology: Animal Behavior Processes, 27, 329-344.

Roberts, A. D. L., \& Pearce, J. (1999). Blocking in the Morris swimming pool. Journal of Experimental Psychology: Animal Behavior Processes, $25,225-235$.

Rodrigo, T., Chamizo, V. D., McLaren., I. P. L., \&. Mackintosh, N. J. (1997). Blocking in the spatial domain. Journal of Experimental Psychology: Animal Behavior Processes, 23, 110-118.

Sovrano, V. A., Bisazza, A., \& Vallortigara, G. (2002). Modularity and spatial orientation in a simple mind: Encoding of geometric and nongeometric properties of a spatial environment by fish. Cognition, 85, B51-B59.

Sovrano, V. A., Bisazza, A., \& Vallortigara, G. (2003). Modularity as a fish (Xenotoca eiseni) views it: Conjoining geometric and non-geometric information for spatial reorientation. Journal of Experimental Psychology: Animal Behavior Processes, 29, 199-210.

Spetch, M. L. (1995). Overshadowing in landmark learning: Touch-screen studies with pigeons and humans. Journal of Experimental Psychology: Animal Behavior Processes, 21, 166-181.

Spetch, M. L., Cheng, K., \& MacDonald, S. E. (1996). Learning the configuration of a landmark array: I. Touch-screen studies with pigeons and humans. Journal of Comparative Psychology, 110, 55-68.

Spetch, M. L., Cheng, K., MacDonald, S. E., Linkenhoker, B. A., Kelly, D. M., \& Doerkson, S. R. (1997). Use of landmark configuration in pigeons and humans: II. Generality across search tasks. Journal of Comparative Psychology, 111, 14-24.

Spetch, M. L., \& Kelly, D. M. (in press). Comparative spatial cognition: Processes in landmark- and surface-based place finding. In E. A. Wasserman \& T. R. Zentall (Eds.), Comparative cognition: Experimental explorations into animal intelligence. Oxford, England: Oxford University Press.

Vallortigara, G., Zanforlin, M., \& Pasti, G. (1990). Geometric modules in animals' spatial representations: A test with chicks (Gallus gallus domesticus). Journal of Comparative Psychology, 104, 248-254.

Wang, R. F., \& Spelke, E. S. (2002). Human spatial representation: Insights from animals. Trends in Cognitive Science, 6, 376-382.

Received September 15, 2003

Revision received March 4, 2004

Accepted March 7, 2004

\section{New Editor Appointed for Journal of Occupational Health Psychology}

The American Psychological Association announces the appointment of Lois E. Tetrick, PhD, as editor of Journal of Occupational Health Psychology for a 5-year term (2006-2010).

As of January 1, 2005, manuscripts should be submitted electronically via the journal's Manuscript Submission Portal (www.apa.org/journals/ocp.html). Authors who are unable to do so should correspond with the editor's office about alternatives:

Lois E. Tetrick, PhD

Incoming Editor, $\mathrm{JOHP}$

George Mason University

Department of Psychology, MSN, 3F5

4400 University Drive, Fairfax, VA 22030

Manuscript submission patterns make the precise date of completion of the 2005 volume uncertain. The current editor, Julian Barling, PhD, will receive and consider manuscripts through December 31,2004 . Should the 2005 volume be completed before that date, manuscripts will be redirected to the new editor for consideration in the 2006 volume. 